\section{Neue Approbationsordnung und Orthopädie}

Seit nunmehr 2 Jahren wir die besonders vom Bundesgesundheitsministerium gewünschte Neufassung der Approbationsordnung für Ärzte heftig diskutiert, wobei Änderungsvorschläge seitens des Ministeriums, der Ländervertreter, des Medizinischen Fakultätentages und der AWMF den gewünschten Konsensus schwierig machten. Es würde den Rahmen dieses Editorials sprengen, auf die Einzelheiten der verschiedenen Entwürfe einzugehen; zeitweilig war die Orthopädie und mit ihr andere sog. ,kleine Fächer“ nicht mehr als ein für alle Studenten prüfungspflichtiges Hauptfach vorgesehen. Nach entsprechender Intervention beim Ministerium, aber auch beim Medizinischen Fakultätentag und insbesondere über die AWMF konnte dies verhindert werden. Der letzte Referentenentwurf vom September 97 wird im Augenblick unter Berücksichtigung nochmaliger Änderungswünsche seitens des Medizinischen Fakultätentages und der AWMF sowie einer Anhörung, an der auch Vertreter der Länder teilnahmen, von den zuständigen Beamten des Gesundheitsministeriums bearbeitet, um bis Januar 98 eine Kabinettsvorlage fertigzustellen. Nach Billigung durch das Kabinett muß schließlich der Bundesrat der Neufassung zustimmen. Sollte dies der Fall sein, könnte die neue Approbationsordnung noch in dieser Legislaturperiode in Kraft treten.

Das in der neuen Approbationsordnung formulierte Ziel der ärztlichen Ausbildung ist der wissenschaftlich und praktisch in der Medizin ausgebildete Arzt, der zur eigenverantwortlichen und selbständigen ärztlichen Berufsausübung, zur Weiterbildung, zu ständiger Fortbildung und zur kritischen Bewertung seines Handelns befähigt ist. Die Ausbildung soll grundlegende Kenntnisse, Fähigkeiten und Fertigkeiten in allen Fächern vermitteln, die für eine umfassende Gesundheitsversorgung der Bevölkerung erforderlich sind. Die Ausbildung zum Arzt wird auf wissenschaftlicher Grundlage durchgeführt und erfolgt durch Hochschullehrer, praxis- und patientenbezogen. Die Vermittlung des theoretischen und klinischen Wissens soll, soweit möglich und zweckmäßig, miteinander verknüpft werden, indem klinische Fächer in den Unterricht des 1. Abschnittes und theoretische Fächer in den des 2. Abschnittes einbezogen werden. Das wie bisher 6 Jahre dauernde Studium besteht aus dem 1. Abschnitt, der zwei Jahre dauert und in dem die klassischen Grundlagenfächer (z. B. Anatomie, Physiologie, Biochemie) gelehrt werden. Hinzu kommen sollen die Molekularbiologie sowie die Grundlagen der medizinischen Psychologie und der medizinischen Soziologie. Um die gewünschte Verknüpfung

Z. Orthop. 136 (1998) 1-2

(c) 1998 F. Enke Verlag Stuttgart der theoretischen und klinischen Fächer zu erreichen, wäre es durchaus denkbar, da $\beta$ beispielsweise der Anatom den Orthopäden in seine Vorlesung einbezieht, wenn er die Anatomie der Bewegungsorgane lehrt. Nach den zwei Jahren erfolgt nunmehr der sog. „Erste Abschnitt der Ärztlichen Prüfung", die schriftlich und mündlich erfolgt und deren Note zu einem Drittel in die spätere Gesamtnote einfließt. Der klinische Teil des Studiums dauert 4 Jahre, das letzte Jahr wie bisher als PJ; danach erfolgt der sog. ,Zweite Abschnitt der Ärztlichen Prüfung“, in dem neuerdings auch schriftlich geprüft wird und zwar in Form von fächerübergreifenden Prüfungsfragen anhand von Fallbeispielen. Der mündliche Teil erfolgt wie bisher in den beiden Hauptfächern „Innere Medizin“ und „Chirurgie" sowie in dem für das PJ gewählte Wahlfach. Die Prüfungsnote wird $\mathrm{zu}^{2}{ }_{3}$ in der Gesamtnote berücksichtigt.

Nach dem 2. Staatsexamen folgt wie bisher die 18-monatige Tätigkeit als AiP, wobei nach Möglichkeit davon $9 \mathrm{Mo}-$ nate in einer nichtoperativen und 6 Monate in einer operativen Abteilung gearbeitet werden soll.

Wichtig für unser Fachgebiet ist die vorgesehene Gestaltung der drei klinischen Jahre nach Bestehen des 1. Abschnittes der ärztlichen Prüfung. Nach der Approbationsordnung kann jede Universität bzw. Fakultät diese Zeit nach eigenem Ermessen strukturieren. Für die Lehre vorgegeben sind über 30 namentlich aufgelistete Fächer -u. a. auch die Orthopädie - und sog. Querschnittsbereiche. Zu den Unterrichtsverantstaltungen gehören neben Vorlesungen insbesondere praktische Übungen sowie Seminare. Als praktische Übungen zählen der Unterricht am Krankenbett, Praktika und Blockpraktika. Der Lehrstoff der klinisch-praktischen Übungen soll sich dabei an den Gegebenheiten der ärztlichen Praxis ausrichten und jeweils nur einer kleinen Gruppe von Studierenden vermittelt werden; bei einer Patientendemonstration sollten höchstens 6 und bei der Untersuchung eines Patienten höchstens 2 Studenten anwesend sein. Soweit Praktika als Blockpraktika durchgeführt werden muß gewährleistet sein, daß pro Studierendem mindestens 10 tagesbelegte Betten mit unterrichtsgeeigneten Patienten zur Verfügung stehen. In den Seminaren sollte der durch praktische Übungen und Vorlesungen vermittelte Lehrstoff vertiefend, anwendungs- und gegenstandsbezogen mit nicht mehr als 20 Studenten erörtert werden. Schließlich können auch noch sog. gegenstandsbezogene Studiengruppen gebildet werden, die einen bestimmten Stoff besprechen und damit eigenständig problemorientiertes Arbeiten üben sollen. Die Prüfungen erfolgen in den 3 klinischen Jahren fächerbegleitend, mündlich und/oder schriftlich. die einzelnen Noten werden in einem Zeugnis aufgelistet, fließen aber nicht in die Staatsexamensnote ein. Dieses Fächerzeugnis ist aber Voraussetzung für die Zulassung zum 2. Abschnitt der ärztlichen Prüfung. 
Da jede Universität die drei klinischen Jahre des Studiums nach ihren eigenen Vorstellungen gestalten darf, besteht die Gefahr, daß der Orthopädieunterricht in Deutschland unterschiedlich intensiv erfolgt. Die orthopädischen Ordinarien müssen sich deshalb frühzeitig in ihrer Fakultät um eine ausreichende Zahl an Unterrichtsstunden bemühen, damit genügend Zeit für Vorlesungen, Praktika und Seminare zugestanden wird. Entsprechend der bekannten großen sozialmedizinischen Bedeutung unseres Faches, der Tatsache, daß über $30 \%$ der Patienten, die einen praktischen Arzt aufsuchen, an Erkrankungen oder Verletzungen der Bewegungsorgane leiden, wäre es dringlich geboten, die Studenten wesentlich intensiver als bisher in der Orthopädie zu unterrichten. Ein wichtiges Ziel der neuen Approbationsordnung ist die Intensivierung des praxisund patientenbezogenen Unterrichtes in Klein- und Kleinstgruppen. Wir sollten diese Chance gerade für unser Fachgebiet nutzen, um in den Praktika und Seminaren mehr als bisher nicht nur unsere wichtigsten und häufigsten Krankheitsbilder darzustellen, sondern auch die Kenntnisse und Fertigkeiten der Studenten in der klinischen Untersuchung der Bewegungsorgane zu vertiefen.
Fundiert in der Orthopädie unterrichtete Studenten werden später als praktische Ärzte z. B. viel eher auf unnötige und teure diagnostische Verfahren verzichten können und ihre Patienten kompetent beraten.

Die neue Approbationsordnung führt zu einer Zunahme der Lehrverpflichtungen, die nicht allein durch die ebenfalls vorgesehene Reduktion der Studierenden um 20\% kompensiert werden kann. Da die Länderminister bereits signalisiert haben, daß die Studienreform kostenneutral bleiben muß und mit der Einführung der neuen Rechtsformen der Universitätsklinika die medizinischen Fakultäten jeweils nur einen bestimmten Betrag für Lehre und Forschung erhalten, wird sich der Verteilungskampf innerhalb der Fakultäten mit Sicherheit verstärken.

Zur Unterstützung unserer Fachvertreter wäre es hilfreich, wenn Ordinarienkonferenz und DGOT einen Mindestkatalog für die Lehre in der Orthopädie erarbeiteten.

L. Jani, Mannheim 